\title{
LDPC Decoding Strategies for Two-Dimensional Magnetic Recording
}

\author{
Anantha Raman Krishnan, Rathnakumar Radhakrishnan, and Bane Vasic, Senior Member, IEEE
}

\begin{abstract}
In this paper, we propose a linear programming (LP) decoding scheme for binary error-erasure channel for use in twodimensional magnetic recording. We compare the performance of this decoding scheme with other decoding schemes like LP decoding for BSC and belief-propagation decoding. Also, we compare the effect of variance of grain-area in the medium on the bit-error rates of various decoding schemes.
\end{abstract}

\section{INTRODUCTION}

The last few years have seen increased research efforts focussed on improving the recording density in magnetic recording systems with densities of upto $10 \mathrm{~Tb} / \mathrm{in}^{2}$ being targetted. Two-Dimensional Magnetic Recording (TDMR) [1] is one of the candidate technologies considered in this regard. TDMR uses conventional media for storing data at high densities while taking an aggressive approach to reading and writing data.

Increasing the recording density in conventional media comes at the expense of reduction in the (average) number of grains used for storing one bit. In fact, at $10 \mathrm{~Tb} / \mathrm{in.}^{2}$, this is as low 1 grain/bit. From a signal processing perspective, this presents many challenges. Firstly, this causes severe two-dimensional (2D) inter-symbol interference (ISI) during readback [1]. Moreover, since conventional media have very irregular grain-boundaries, making the bit-area comparable to grain-area in such media leads to a high degree of irregularities in the bit-boundaries. This is a significant source of noise during readback. Indeed, the signal-to-noise ratio (SNR) can be as low as $0 \mathrm{~dB}$ [1]. It has also been observed that the noise is highly correlated in both the "down-track" and "crosstrack" direction [2], [3]. These factors prompt the need to develop sophisticated 2D signal processing algorithms in order to achieve the goal of reliable data storage at such high recording densities.

The strategy proposed for achieving low bit-error rates (BER) in TDMR is to first achieve reasonably low detected biterror rates (BER) (say, of the order of $10^{-2}$ ) and rely on strong (comparatively lower rate) error-correcting codes to further reduce the BER [1]. There has been considerable progress in read-channel modelling and detector design [2], [3], [4]. Previously, design of detectors that achieve BER of about $5 \times 10^{-2}$ have been reported [3]. However, design of errorcontrol systems for TDMR has been relatively unexplored.

The use of low-density parity-check (LDPC) codes [5] for magnetic recording have been proposed on account of their good performance [6], [7], and are a good choice for

A. R. Krishnan, R. Radhakrishnan and B. Vasic are with the Department of Electrical and Computer Engineering, The University of Arizona, Tucson, AZ, 85721 USA e-mail: \{ananthak, rathna, vasic\}@ece.arizona.edu.
TDMR. Traditionally, belief-propagation (BP) decoders [5], [8] have been used for decoding LDPC codes. Another type of decoding decoder that has received much attention recently is the linear programming (LP) decoding of binary linear codes [9]. LP decoding have been considered as a suboptimal alternative to ML decoding. Their performance and the fact that they possess the ML certificate makes them appealing. Also, the nature of the decoding algorithm makes them more amenable to analysis (see [9] for more details), thereby making them potential candidates for decoders with predictable performance.

In this paper, we propose a method to transform the TDMR channel into a binary error-erasure channel (BEEC) and use LP decoding for BEEC to correct errors and erasures. As a proof of concept, we compare the performance of this scheme to that of other well-known decoding schemes. Also, we compare the effect of improving the regularity of grains on different decoding schemes. The rest of the paper is organized as follows: Section II explains the system model in which we briefly describe the model of the recording medium and the readback process. In Section III, we present detector architecture for the TDMR channel. An LP decoder for the TDMR channel is presented in Section IV. We then compare this decoder to other existing decoders in Section V. Finally, we summarize our results in Section VI.

\section{SySTEM MODEL}

Figure 1 shows the schematic representation of the TDMR read channel. As shown in the diagram, the irregularity in the recording medium source of noise. Since electronic noise is very less compared to this noise, we assumed it to be zero. The errors and interference in the system are compensated by detection and decoding (to be discussed in detail).

The modelling of TDMR read-channel involves modelling (1) the recording medium, (2) the write process, and (3) the readback process. Although this has been discussed in detail in [2], we give a brief description of the model for the sake of completeness, and as it forms a basis for detector and decoder design.

\section{Recording Medium Model}

Let $\mathcal{S}$ be the set of points on a square lattice. The ideal medium (from the perspective of the detector) is modelled as the Voronoi tiling of points in $\mathcal{S}$ with their Voronoi regions representing the grains. We refer these points as cell-centers and their corresponding Voronoi regions as cells. Fig. 2(a) shows an ideal medium of dimension $3 \times 3$ cells with the cellcenters marked as ' $*$ ' and cell boundaries marked with dotted lines. 


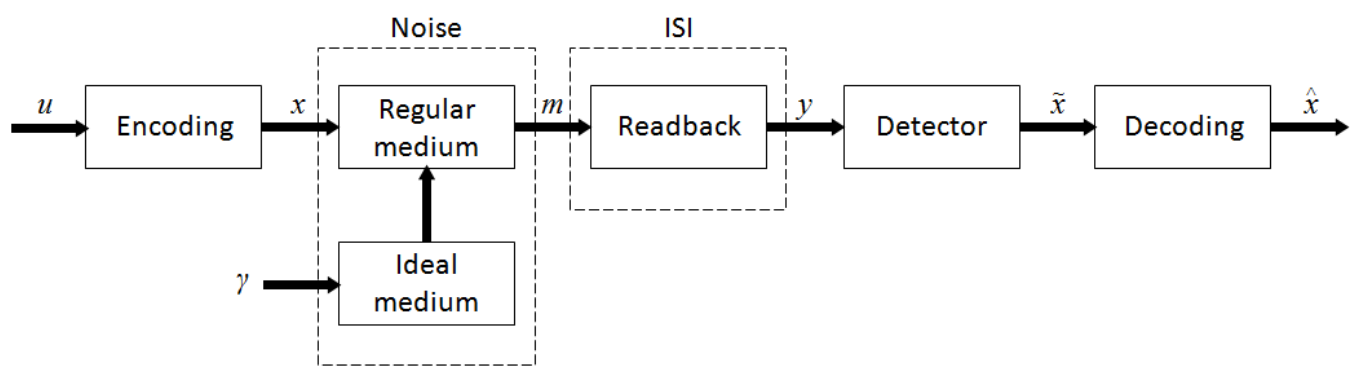

Fig. 1. Schematic representation of the TDMR read channel model

A regular medium is obtained by first randomly shifting the points in $\mathcal{S}$ and then finding the Voronoi tiling of the shifted points. The shifted points are referred to as graincenters. Fig. 2(b) shows an example of a non-ideal recording medium with the grain-centers and cell-centers marked as ' $\bullet$ ' and '*', respectively. The grain-boundaries and cell-boundaries are marked with solid and dotted lines, respectively.

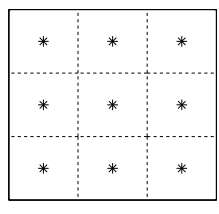

(a)

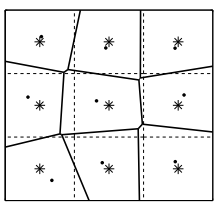

(b)
Fig. 2. Modelling of recording medium: (a) an example of an ideal medium, and (b) an example of a non-ideal medium.

For modelling the shift of grain-centers, Tikhonov distribution [10], [11] scaled between -0.5 and +0.5 is used. The probability density function (pdf) for Tikhonov distribution is given as:

$$
p_{\gamma}(\Delta x)=\frac{\exp (\gamma \cos (\Delta x))}{2 \pi I_{0}(\gamma)} 0 \leq \Delta x \leq 2 \pi,
$$

where $I_{0}$ is the zeroth order modified Bessel function of the second kind. The parameter $\gamma$ varies from 0 to $\infty$, and describes the level uniformity in the grain sizes in the recording medium. When $\gamma=0, \Delta x$ is uniformly distributed in $[-\pi, \pi]$. When $\gamma=\infty, p_{\infty}(\Delta x)=\delta(\Delta x)$ ( $\delta$ is the Dirac delta function). Fig. 3 shows the pdfs for various values of $\gamma$.

Figures 4(a) and 4(b) show instances of recording media for $\gamma=0$ and 4 , respectively. It can be easily seen that the medium shown Fig. 4(b) is more regular in grain shape and position than the medium shown in Fig. 4(a). Fig. 5 shows the variance of grain-area for various values of $\gamma$. We note that the grain variance is the highest for $\gamma=0$ and approaches zero as $\gamma$ tends to $\infty$.

\section{A. Write and Readback process}

Since the read/write mechanism does not have a priori knowledge of the positions of the grains in the medium, it simply attempts to write at the center of each cell. The grain whose center is within the cell boundary is magnetized appropriately. The readback signal is obtained as a convolution of the magnetization of the recording medium with the $2 \mathrm{D}$ impulse response of the read-head. We assume the impulse response to be a truncated Gaussian window of unit energy with a half-maximum width of 1 cell and a span of 3 cells in both dimensions.

\section{TDMR DETECTION}

Let $\mathcal{C}=\left\{\left(c_{1}, c_{2}\right): c_{1}, c_{2} \in\{0,1, \ldots, n-1\}\right\}$ be the points on an $n \times n$ square lattice that define the positions of cell-centers. Let $\mathbf{X}=\left\{x_{0,0}, x_{0,1}, \ldots, x_{0, n-1}, x_{1,0}, \ldots, x_{n-1, n-1}\right\} \quad \in$ $\{-1,+1\}^{n^{2}}$ be the input magnetization and let $\mathbf{Y}=\left\{y_{0,0}, y_{0,1}, \ldots, y_{0, n-1}, y_{1,0}, \ldots, y_{n-1, n-1}\right\} \in \mathbb{R}^{n^{2}}$ be the readback signal. Let $m\left(t_{1}, t_{2}\right)$ be the magnetization of the recording medium at the point $\left(t_{1}, t_{2}\right)$. Let $A_{i, j}$ be the region spanning the $(i, j)^{t h}$ cell. Then, the readback signal, $y_{i, j}$, at center of the $(i, j)^{t h}$ cell is given as:

$$
\begin{aligned}
y_{i, j}= & \left.y\left(t_{1}, t_{2}\right)\right|_{(i, j)} \\
= & \iint h\left(\tau_{1}, \tau_{2}\right) x\left(i-\tau_{1}, j-\tau_{2}\right) \mathrm{d} \tau_{1} \mathrm{~d} \tau_{2} \\
= & \sum_{k_{1}=-1}^{+1} \sum_{k_{2}=-1}^{+1} \\
& \iint_{A_{k_{1}, k_{2}}} h\left(\tau_{1}, \tau_{2}\right) x\left(i-\tau_{1}, j-\tau_{2}\right) \mathrm{d} \tau_{1} \mathrm{~d} \tau_{2} .
\end{aligned}
$$

In an ideal medium, the grain boundaries coincide with the bit-boundaries. Hence, we have:

$$
m\left(\tau_{1}, \tau_{2}\right)=x_{\hat{\tau}_{1}, \hat{\tau}_{2}}
$$

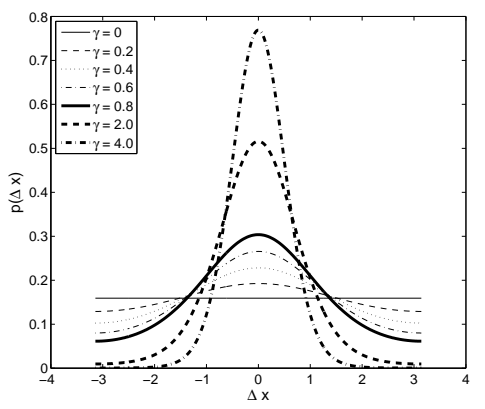

Fig. 3. Tikhonov distribution: The pdfs for various values of $\gamma$ 


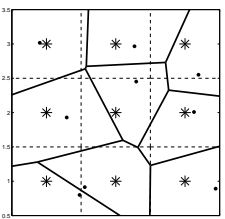

(a)

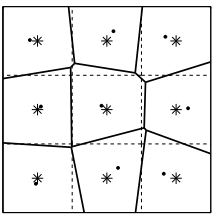

(b)
Fig. 4. Instances of recording media for different values $\gamma$ 's: (a) $\gamma=0$; and (b) $\gamma=4$. The cell-centers and the grain-centers are denoted by "**" and "•", respectively. The cell-boundaries and grain-boundaris are marked with dashed and solid lines, respectively.

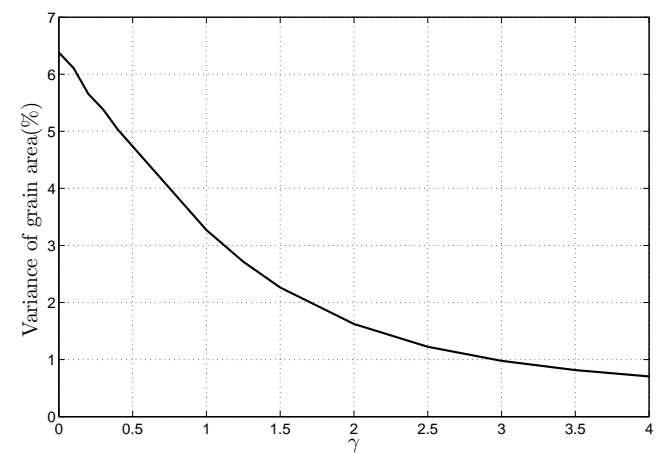

Fig. 5. Variance of grain area for various values of $\gamma$.

where $\hat{\tau}$ is $\tau$ rounded to the nearest integer and is given as $\hat{\tau}=\lfloor\tau+0.5\rfloor$. Hence, the signal equation for an ideal medium can be written as

$$
y_{i, j}=\sum_{k_{1}=-1}^{+1} \sum_{k_{2}=-1}^{+1} h_{k_{1}, k_{2}} x_{i-k_{1}, j-k_{2}}
$$

where,

$$
h_{k_{1}, k_{2}}=\iint_{A_{k_{1}, k_{2}}} h\left(\tau_{1}, \tau_{2}\right) \mathrm{d} \tau_{1} \mathrm{~d} \tau_{2} .
$$

That is, in an ideal medium, the output signal can be expressed as a discrete convolution of the input magnetization and the discretized read-head response. Consider now the detector that is a zero forcing equalizer with impulse response $h^{-1}$. Then, for an ideal medium with no electronic noise, this is the optimal detector. Indeed, the detected output, $\tilde{x}_{i, j}=\left(h^{-1} * x\right)_{i, j}$, is equal to input $x_{i, j}$. Suppose this zeroforcing detector, which assumes the the medium to always be ideal, is used to detect input magnetization on a non-ideal medium, then we have

$$
\tilde{x}_{i, j}=\left(h^{-1} * x\right)_{i, j},
$$

where $\tilde{x}_{i, j} \in \mathbb{R}$. We term $\tilde{x}$ as the effective magnetization. Effective magnetization can also be interpreted as a noisefree ISI channel with real inputs, $\tilde{x}$. Fig. 6 shows the $p(\tilde{x} \mid x)$ obtained experimentally. We emphasize here that in general, the effective magnetization are correlated in both dimensions. However, as a simplification, we assume that they are uncorrelated.

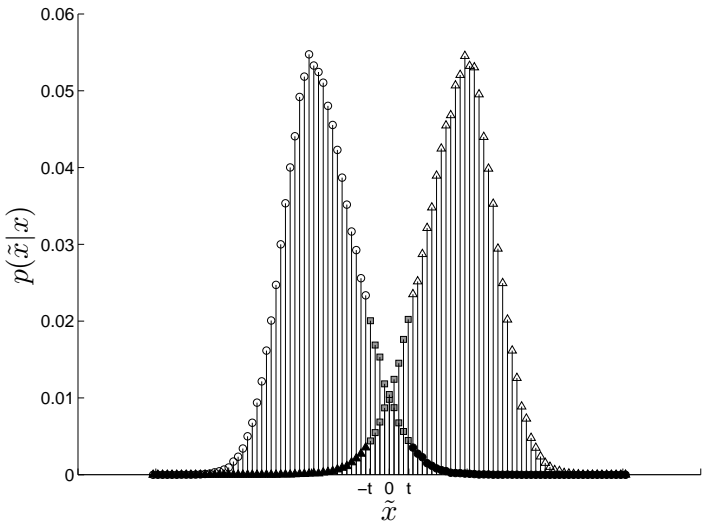

Fig. 6. Pdf of the $\tilde{x}$ given the input magnetization. The squares denote erasures. Empty cicles and triangles represent correctly detected magnetization and filled squares and circles represent magnetization which are incorrectly decoded.

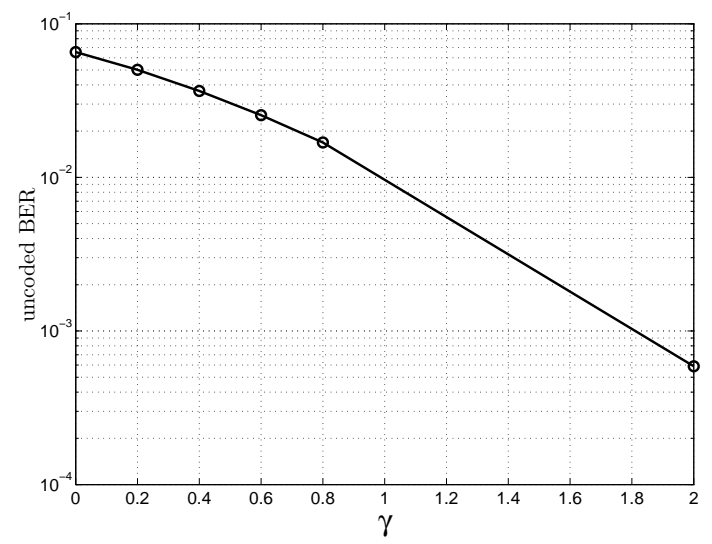

Fig. 7. Plot of uncoded BER versus $\gamma$.

Using this detection scheme, uncoded data can be detected by simply thresholding the effective magnetization based on its sign.

Error rates of uncoded data: In order to test the effect of variance of grain size on the uncoded bit-error rate, we generated recording media with the shifted grain-centers following the Tikhonov distribution for different values of $\gamma$. The $\gamma$ 's chosen were $0.2,0.4,0.6,0.8$ and 2 . Fig. 7 shows a plot comparing the uncoded BER with $\gamma$. It can be seen that there is significant decrease in BER with only slight increase in $\gamma$ (i.e., reduction in variance of grain-size).

We note that for coded data, the continuous nature of $\tilde{x}$ allows us to process it in a number of ways, thereby providing us with a choice from a number of novel decoding architectures, each varying in complexity and performance. Consider the following transformation that maps $\tilde{x}$ to elements of the set $\{-1,0,+1\}$ [3] given by:

$$
\hat{x}_{i, j}=\left\{\begin{aligned}
1 & \text { if } \tilde{x}_{i, j} \geq t \\
-1 & \text { if } \tilde{x}_{i, j} \leq-t \\
0 & \text { if }-t<\tilde{x}_{i, j}<t .
\end{aligned}\right.
$$

where $t \geq 0$. This way, we can transform the TDMR channel into a binary error-erasure channel (BEEC) (where the symbol 
0 indicates an erasure). The probabilities of error $(p)$, and the probability of erasure $(\epsilon)$ of this BEEC is given by:

$$
\begin{aligned}
p & =\int_{\tilde{x} \geq t} p(\tilde{x} \mid x=-1) \mathrm{d} \tilde{x}+\int_{\tilde{x} \leq-t} p(\tilde{x} \mid x=+1) \mathrm{d} \tilde{x}(1) \\
\epsilon & =\int_{|\tilde{x}|<t} p(\tilde{x} \mid x=-1) \mathrm{d} \tilde{x}+\int_{|\tilde{x}|<t} p(\tilde{x} \mid x=+1) \mathrm{d} \tilde{x}
\end{aligned}
$$

The regions of the histogram corresponding to each of the regions is shown in Fig. 6. In the figure, the region marked with filled circles and filled triangles denote bits detected incorrectly. The region marked with grey squares denotes the bits detected as erasures. It can be seen that for different values of $t$, we get different values for $p$ and $\epsilon$. For $t=0$, the channel is equivalent to a binary symmetric channel (BSC).

\section{LDPC CODES FOR ERRORS AND ERASURES}

As seen before, the TDMR channel is easily transformed into a binary error and erasure channel. However, in the absence of demonstrable LDPC decoders capable of correcting errors and erasures, such a transformation is of little significance. LDPC decoding for BEEC was independently conceived by Mitzenmacher [12] and Richardson et al. [13]. They proposed hard-input/hard-output message-passing algorithm for this channel. Apart from this work, LDPC decoders for BEEC have been largely unexplored. In this paper, we apply LP decoding for the first time to BEEC. Before we describe this, we briefly describe LP decoding [9]. we use notations and explanation from [9].

Let $\mathcal{C}$ be a binary linear code with length $n$ with its paritycheck matrix $H$. A binary word $c=\left(c_{1}, c_{2}, \ldots, c_{n}\right)$ is a codeword if and only if $c H^{T}=0$. Define the codeword polytope $\operatorname{poly}(\mathcal{C})$ to be the polytope whose vertices are the codewords of $\mathcal{C}$. Assume that $c$ is transmitted through a symmetric memoryless channel. Let $\hat{c}=\left(\hat{c}_{1}, \hat{c}_{2}, \ldots, \hat{c}_{n}\right)$ be the received vector. Then, ML decoding of $\hat{c}$ is equivalent to the calculation of the vector $f=\left(f_{1}, f_{2}, \ldots, f_{n}\right)$ which minimizes $\sum_{i=1}^{n} g_{i} f_{i}$, where $g_{i}$ is the negative log-likelihood ratio (LLR) of the $i^{\text {th }}$ variable and is given as:

$$
g_{i}=\log \left(\frac{P\left(\hat{c}_{i} \mid c_{i}=0\right)}{P\left(\hat{c}_{i} \mid c_{i}=1\right)}\right)
$$

subject to the constraint $f \in \operatorname{poly}(\mathcal{C})$. However, this is impractical as the number of constraints is exponential in $n$. Hence, we define a relaxed polytope, which is an intersection of local polytopes defined at each parity-check. For each parity-check $j$, let $N(j)$, denote the set of variables connected to $j$. Let $E(j)=\{S \subseteq N(j):|S|$ even $\}$. The local polytopes, $Q_{j}$, of the $j^{t h}$ check is the set of points $(f, w)$ that satisfy the following constraints:

$$
\begin{gathered}
\text { for } 1 \leq i \leq n, 0 \leq f_{i} \leq 1 \\
\forall S \in E(j), 0 \leq w_{j, S} \leq 1 \\
\sum_{S \in E(j)} w_{j, S}=1 \\
\forall i \in N(j), \quad f_{i}=\sum_{\substack{S \in E(j) \\
S \ni i}} w_{j, S}
\end{gathered}
$$

The relaxed polytope is defined as $Q=\bigcap_{j} Q_{j}$. The Linear Code Linear Program decoder is the argument $(f, w)$ that solves the following linear program:

$$
\text { minimize } \sum_{i} g_{i} f_{i} \text { s.t. }(f, w) \in Q
$$

For the BEEC with probability of error $p$ and probability of erasure $\epsilon, g_{i}$ is defined as (henceforth, we refer to the input and the output alphabet of the BEEC as $\{0,1\}$ and $\{0, e, 1\}$, respectively.):

$$
g_{i}=\left\{\begin{aligned}
-L & \text { if } \hat{c}_{i}=0 \\
+L & \text { if } \hat{c}_{i}=1 \\
0 & \text { if } \hat{c}_{i}=e
\end{aligned}\right.
$$

where $L=\log (p /(1-p))$. By changing the $g_{i}$ thus, LP decoding of errors and erasures can be performed. We note here that for each integral solution $(f, w)$, there exists a codeword of $\mathcal{C}$. Hence, the decoding is said to be successful whenever there is an integral solution. A decoder failure occurs when one or more elements of $(f, w)$ are non-integers. In our experiments, we simply threshold the values of $f_{i}$ to 0 or 1 in the case of a decoder failure.

\section{Simulation Results And Discussion}

In this section, we compare the performance of LDPC codes under LP decoding for BEEC with some other wellknown decoding strategies, namely, LP decoding for BSC (as explained above), and BP decoding for coninuous(soft)-output channel [5], [8]. For our analysis, we chose a code of length 155 with girth 8 and rate 0.4 constructed using a modified version of the progressive edge-growth algorithm [14]. This construction technique yields codes with guaranteed errorcorrection performance under iterative decoding for BSC (see [14] and reference therein for details). Such codes have been well-studied theoretically and analytically, and their properties well-understood. Also, choosing short-length codes helps us in identification and analysis of error-events in TDMR. We wish to emphasize that that no attempt at optimizing the code for the TDMR channel was made as the experiments are intended only as proof of concept.

For our analysis, the pdfs $p(\tilde{x} \mid x)$ were obtained experimentally for each $\gamma$. The probabilities of error (for LP decoding) were obtained from the histograms by using the Eqn. 1. For the BP decoder, the log-likelihood ratios were obtained from the histograms as $\log (p(\tilde{x} \mid x=+1) / p(\tilde{x} \mid x=-1))$.

Fig. 8 shows the performance of LP decoding for BEEC for various values of threshold $t$ in media with $\gamma=0$ (i.e., media with very high variance in grain area). Note that the BER for $t=0$ corresponds to decoding in BSC. It can be seen that for values of $t$ between 0 and 0.35 , the BER reduces with increase in $t$. This is because, for these values of threshold, the BEEC detector benefits from the reduction in error-rate. However, at high values of $t$, the average number of erasures is very high, and hence is not corrected by the decoder. In general, the values of $t$ that yield the best results depend on $\gamma$. We choose 0.25 which is typically within this range.

Fig. 9 compares the performance of LP decoding for BEEC in media with different $\gamma$ with those of LP decoding for BSC 


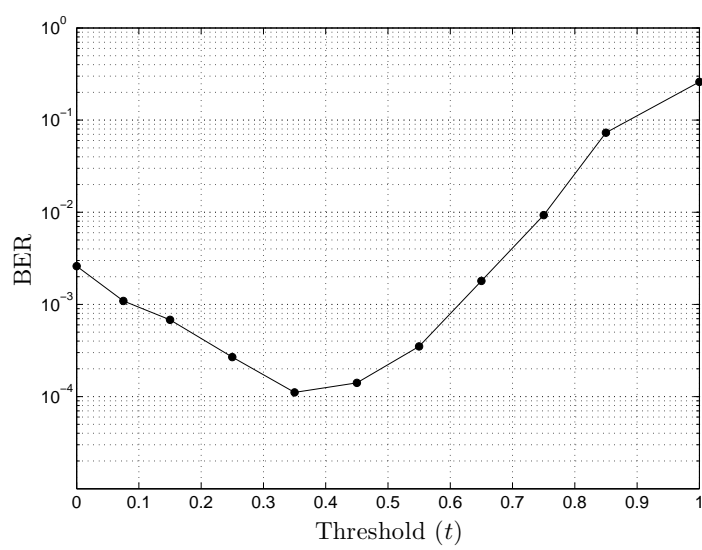

Fig. 8. BER for various values of threshold

and BP decoding. The number of decoding iterations for the BP decoder is 100 . It can be seen that for small values of $\gamma$, LP decoding for BEEC performs slightly worse than for BP decoding. However, at higher values of $\gamma$, it outperforms BP decoding. We also observe that as in the case of uncoded data, large improvements in performance of coded detection is possible through a very small reduction the variance of grainarea. In particular, by reducing the variance in grain-area from $6.3 \%$ to $5.3 \%$, the BERs of the decoding schemes improve by about 2 orders of magnitude.

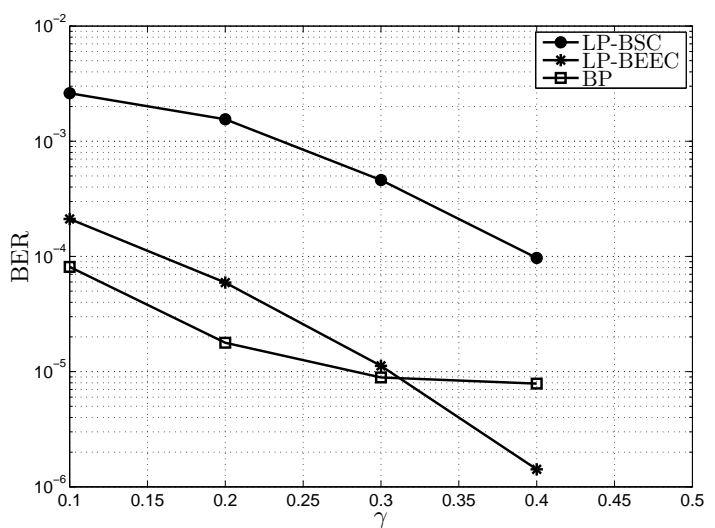

Fig. 9. Plot of $\gamma$ versus BER for various decoding schemes.

It must be noted that the BER plot for BP decoding indicates the presence of an error-floor at a BER of around $10^{-5}$. However, performance of this code in AWGN does not suffer from any error-floors, as is seen in Fig. 10. Although it is not fully understood at this time, we hypothesize that the degradation in the performance of BP in TDMR channel is due to the high correlation among the noise samples, which is not accounted for during detection/decoding.

\section{CONCLUSION}

In this paper, we proposed an LP decoding architecture for BEEC as an alternative to BP decoding in TDMR. Experimentally, we showed that LP decoding for BEEC outperforms

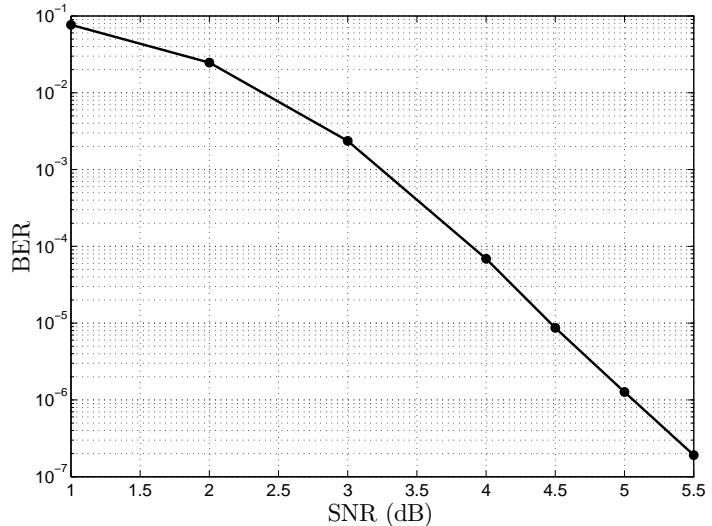

Fig. 10. Performance of the code on AWGN channel.

LP decoding for BSC. Their performance has been shown to be comparable to that of BP decoding. Also, we showed that by enforcing slight control over the regularity of the recording medium, large improvements in BERs can be obtained. Future work in this direction include analysis of error-events for the LP decoder and code-design targetted at improving the performance of LDPC codes in the TDMR channel.

\section{REFERENCES}

[1] R. Wood, M. Williams, A. Kavcic, and J. Miles, "The feasibility of magnetic recording at 10 terabits per square inch on conventional media," in Proc. of The Magnetic Recording Conference, Jul. 2008.

[2] A. R. Krishnan, R. Radhakrishnan, and B. Vasic, "Read-channel modeling for detector design of two-dimensional magnetic recording systems," submitted to IEEE Trans. Magn.

[3] B. Vasic, A. R. Krishnan, R. Radhakrishnan, A. Kavcic, W. Ryan, and F. Erden, "Two-dimensional magnetic recording: read channel modeling and detection," submitted to IEEE Trans. Magn.

[4] S. C. Kheong et al., "TDMR platform simulations and experiments," submitted to IEEE Trans. Magn., 2009.

[5] R. G. Gallager, Low Density Parity Check Codes. Cambridge, MA: M.I.T. Press, 1963.

[6] J. Fan, E. Kurtas, A. Friedman, and S. McLaughlin, "low-density paritycheck codes for magnetic recording," in Proc. 37th Allerton Conf., 1999, pp. 1314-1323.

[7] M. Yang and W. E. Ryan, "Performance of efficiently encodable lowdensity parity-check codes in noise bursts on the epr4 channel," IEEE Trans. on Magn., vol. 40, no. 2, Mar. 2004.

[8] F. R. Kschischang, B. J. Frey, and H.-A. Loeliger, "Factor graphs and the sum-product algorithm," IEEE Trans. on Inf. Theory, vol. 47, no. 2, pp. 498-519, Feb. 2001.

[9] J. Feldman, M. J. Wainwright, and D. R. Karger, "Using linear programming to decode binary linear codes," IEEE Trans. on Inf. Theory, vol. 51, no. 3, pp. 954-972, 2005.

[10] H. L. V. Trees, Detection, Estimation, and Modulation Theory, Part I. New York: Wiley, 1968.

[11] T. Eng and L. B. Milstein, "Partially coherent ds-ss performance in frequency selective multipath fading," IEEE Trans. on Comm., vol. 45 , no. 1 , pp. $110-118,1997$.

[12] M. Mitzenmacher, "A note on low-density parity-check codes for erasures and errors," SRC Technical Note, 1998.

[13] T. J. Richardson and R. L. Urbanke, "The capacity of low-density paritycheck codes under message-passing decoding," IEEE Trans. on Inf. Theory, vol. 47, no. 2, pp. 599-618, 2001.

[14] S. K. Chilappagari, A. R. Krishnan, and B. Vasic, "LDPC codes which can correct three errors under iterative decoding," in Proc. of Information Theory Workshop (ITW), May 2008. 